\title{
Review of: "Role of minimally invasive glaucoma surgery in the management of chronic open-angle glaucoma"
}

Daniel Laroche, Abelard Desrosiers ${ }^{1}$

1 City University of New York

Potential competing interests: The author(s) declared that no potential competing interests exist.

Review of Role of minimally invasive glaucoma surgery in the management of chronic open-angle glaucoma

Daniel Laroche MD

Clinical Assistant Professor of Ophthalmology, New York Eye and Ear Infirmary of Mount Sinai Abelard Desrosiers BS

MS2, CUNY School of Medicine

This is a nice retrospective comparative case series study done in a single hospital in the United Kingdom that compared the safety and efficacy of phacoemulsification combined with ab-interno trabeculectomy (Trabectome) and phacoemulsification combined with I-Stent inject(Pi). Consecutive patients with medically uncontrolled primary open-angle glaucoma (POAG) were followed for 2 years after undergoing Trabectome or Pi done by a single surgeon in 2017-2018. Efficacy and safety were measured during the two year period by looking at the Mean IOP, percentage of patients using eye drops, mean number of eye medications, surgical success criteria, and change in vision. The principal findings in this study were that there was excellent lowering of IOP in both the Pi group and the Trabectome group both showing a $20 \%$ drop in IOP. The success rate (target IOP achieved and maintained for 2 years) in advance glaucoma was higher in the Pi group (30.7 vs $25 \%$ ) and the success rate in mild to moderate was higher in the Pi group compared to the Trabectome group (90\% vs $85.71 \%$ ). Both surgical interventions were found to be safe and efficacious, however those in the Pi group had slightly better outcomes and both procedures are less effective treatments for patients with advanced glaucoma. Most of the patients in this study have favorable surgical outcomes with a small percentage have cystoid macular edema that eventually resolved except for one patient with residual decreased vision.

The limitations in this study are the following: 
1. This study has retrospective nature of the data collection, lack of randomization, and potential bias in choosing one technique over another.

2. There was not a cataract surgery alone group to compare. We know that cataract surgery alone can lower IOP about $5 \mathrm{mmHG}$. All the patients in this study did have a greater IOP lowering than $5 \mathrm{mmgHG}$ since all the cataract surgeries were combined with a MIGS procedure.

3. There is no discussion of post operative IOP spikes and management. This is a well known anticipated potential complication that has to be managed whether discussing retained viscoelastic, hyphema and keeping the patient's head elevated postoperatively for a few days to reduce hyphema, steroid IOP spikes, and tapering. The authors did use Pilocarpine post-operatively that may have mitigated some of IOP spikes seen post-operatively.

4. The age range of patients in this study are all in the 8th decade of life. Although patients who are diagnosed with POAG are typically elderly, patients over the age of 40 are at an increased risk for developing POAG. Given that increased risk, it has been predicted that over the next decade that number of people diagnosed with POAG between the ages of 40-80 will increase. As a result a significant percentage of patients had more advanced glaucoma. It will be interesting to see if earlier cataract surgery/refractive lensectomy and MIGS in younger patients will have even better results and reduce the risk for the need for trabeculectomy. This has been seen in more recent MIGS studies. ${ }^{1}$

5. There is no pre or post op visual field notations to confirm visual field preservation particularly in the mild to moderate group. Visual field preservation has been demonstrated with the Hydrus stent both short term and with recent 5 year data. ${ }^{2}$

6. We agree with the authors that Early Cataract surgery and MIGS are very important in lowering IOP in early and moderate glaucoma. In fact this has become our initial approach to glaucoma management. We prefer to do this before medical therapy and or SLT to preserve the distal outflow of the aqueous drainage system. Our preferred MIGS is the Hydrus stent.

7. The authors acknowledge that the cost of MIGS are expensive. In resource poor areas this can also be performed with a straight bent cystotome, 23G, 25G, or 27G. This can also be performed with a sinsky hook and MVR blade. ${ }^{3,4}$

\section{References}

1. https://www.prnewswire.com/news-releases/hydrus-microstent-lowers-visual-field-loss-by-47-accordingto-new-5-year-pivotal-trial-data-301423785.html

2. Laroche, D., Nkrumah, G., \& Ng, C. (2020). Real-world efficacy of the Hydrus microstent in Black and Afro-Latinx patients with glaucoma: a retrospective study. Therapeutic Advances in Ophthalmology, 12, 251584142096431. https://doi.org/10.1177/2515841420964311

3. Laroche, D., Okaka, Y., \& Ng, C. (2019). A Novel Low Cost Effective Technique in Using a 23 Gauge 
Straight Cystotome to Perform Goniotomy: Making Micro-invasive Glaucoma Surgery (MIGS) Accessible to the Africans and the Diaspora. Journal of the National Medical Association, 111(2), 193-197. https://doi.org/10.1016/j.jnma.2018.09.006

4. https://www.youtube.com/watch?v=pJUfULz62Xo 